\title{
Seeing is as Good as Doing
}

\author{
Magda Osman ${ }^{1}$
}

\begin{abstract}
Given the privileged status claimed for active learning in a variety of domains (visuomotor learning, causal induction, problem solving, education, skill learning), the present study examines whether action-based learning is a necessary, or a sufficient, means of acquiring the relevant skills needed to perform a task typically described as requiring active learning. To achieve this, the present study compares the effects of action-based and observation-based learning when controlling a complex dynamic task environment $(\mathrm{N}=$ 96). Both action- and observation-based individuals learn either by describing the changes in the environment in the form of a conditional statement, or not. The study reveals that for both active and observational learners, advantages in performance $(p<.05)$, accuracy in knowledge of the task $(p<.05)$, and self-insight $(p<.05)$ are found when learning is based on inducing rules from the task environment. Moreover, the study provides evidence suggesting that, given task instructions that encourage rule-based knowledge, both active and observation-based learning can lead to high levels of problem solving skills in a complex dynamic environment.
\end{abstract}

\section{Seeing is as Good as Doing}

Who has better knowledge and skill: the back seat driver, who is learning to drive, or the actual driver, who is also learning to drive; the person watching their friend play a new game on the Sony play station, or the friend who is actually playing the game? Our daily lives frequently involve learning to control complex dynamic environments like those referred to in the question, but how we come to form the relevant skills needed to master such environments remains much debated. Laboratory versions of these tasks, referred to as Complex dynamic control tasks (CDC-tasks; see Figure 1: water purification system) typically include several inputs (salt, carbon, lime) that are connected via a complex structure or rule to several outputs (chlorine concentration, temperature, oxygenation).

\footnotetext{
${ }^{1}$ University College London

Address correspondence to:

Magda Osman, Department of Psychology, University College London, Gower Street, London WC1E 6BT, England

Telephone:+4420 7679 7572; Fax:+4420 7436 4276; Email:m.osman@ucl.ac.uk
} 
In such environments, people are required to make a series of decisions, often in real time, that each depend on the other, and in an environment that changes autonomously as a consequence of a person's actions (Brehmer, 1992).

Implicit theorists (Berry, 1991; Berry \& Broadbent, 1984, 1987, 1988; Dienes \& Berry, 1997; Lee, 1995; Stanley, Mathews, Buss, \& Kotler-Cope, 1989) claim that the learning process involved in dynamic task environments is procedural. The knowledge that is acquired is "knowing how" to perform actions that are tied to specific goals. In contrast, declarative knowledge, which is "knowing that" of particular facts about the underlying actions, involves structural knowledge concerned with the goal itself (Anderson, 1982). By extension, implicit learning theorists (Berry, 1991; Berry \& Broadbent, 1988; Dienes \& Berry, 1997; Lee, 1995; Sun, Merrill, \& Peterson, 2001) have proposed that knowledge acquired in CDC-tasks and experience in controlling them is procedural and embedded within the interactions problem solvers have with the system. This form of learning produces instance-based procedural knowledge, that is, specific actions undertaken while interacting with the system become associated with the specific effects that they generate. Thus, mastering a control task requires successfully matching the goal and the current situation to previously encountered instances in order to determine the next appropriate response. Knowledge is conscious only to the extent that the response appropriate to a given situation can be stated, but what led them to make this response is unavailable to consciousness (Buchner, Funke, \& Berry, 1995; Dienes \& Berry, 1997; Dienes \& Fahey, 1995, 1998).

The empirical foundation of this position is the phenomenon showing that declarative knowledge is dissociated from procedural knowledge. This is because the learning systems understood to support declarative and procedural knowledge are distinct. In support of this, people have been shown to successfully control a CDC-task independently of any reportable declarative knowledge of the rule or causal structure that determines its operation, and without self-insight as to how they are able to perform it (Berry \& Broadbent, 1984, 1987, 1988; Stanley et al., 1989). Another compelling demonstration of dissociations between rule- and instance-based knowledge is found in the contrasting effects of observation-based and procedural-based learning (Berry, 1991; Lee, 1995). Because declarative knowledge cannot easily be used to execute tasks suited to procedural knowledge (Berry, 1991; Berry \& Broadbent, 1984, 1987, 1988; Dienes \& Berry, 1997; Lee, 1995), it has also been claimed that possessing declarative knowledge will impair one's later ability to perform a procedural task. Observation-based learning involves problem solvers watching the actions taken by another problem solver attempting to learn a CDC-task. This encourages the observer to focus on understanding how the system operates (i.e., rule-based knowledge), and is claimed to engage the explicit learning system (Berry, 1991). In contrast, conventional methods of training to control a CDC-task are procedural based and are thought to encourage knowledge of how to operate the system (i.e., instancebased knowledge). When compared, observers show better rule-based knowledge than 
procedural learners, but poorer control performance, further demonstrating dissociations between procedural and declarative learning.

Others, however, have suggested that successful skill acquisition depends on a combination of rule-based and instance-based knowledge, which develops through hypothesis testing (Burns \& Vollmeyer, 2002; Osman, 2008a, 2008b; Sweller, 1988; Vollmeyer, Burns, \& Holyoak, 1996). By exploring the task and formulating rules about how it operates, learners are able to update their rule-based knowledge through the instances that they have generated to test them. Through practice, a wider range of instances are experienced, and these enable learners to form generalizable knowledge that they can transfer to other similar tasks. Evidence for this comes from studies that compare different types of goal instructions during learning. For instance, instructions like "explore the system," an example of a nonspecific goal (NSG), are contrasted with "learn about the system while trying to reach and maintain specific outcomes," an example of a specific goal (SG). The former instruction is assumed to encourage hypothesis testing, because rules can be generated and tested, whereas in the latter instruction, learning is constrained by generating instances that fulfill specific criteria. When tested on their ability to control the system to previously trained goals, SG-learners' performance is equal to that of NSG-learners that have not learned to perform the task to any criteria. Furthermore, for untrained goals, NSG-learners outperform SG-learners. Taken together with evidence that NSG-learners also have superior structural knowledge about the system, this suggests that rule- and instance-based knowledge combined is more effective than instance-based knowledge alone (Burns \& Vollmeyer, 2002; Osman, 2008a; Vollmeyer et al., 1996).

Given these conflicting views, this article asks: Is procedural learning necessary to ensure skill acquisition in a complex dynamic environment? To address this, the present study included six conditions (Active [generate]-Instance, Active [generate]-Instance + Rule, Active [replicate]-Instance, Active [replicate]-Instance + Rule, Observe-Instance, ObserveInstance + Rule), across which the involvement of procedural learning was gradually attenuated. If procedural learning is necessary for skill development in CDC-tasks, then active-learning conditions will consistently show superior control performance compared with observation-based learning conditions, but poorer rule-based knowledge. If instead, instance- and rule-based knowledge combined are necessary for control skills to develop, then, regardless of action- or observation-based learning, both kinds of learners will show superior knowledge relative to those acquiring only instance-based knowledge.

\section{Method}

Ninety-six students from University College London volunteered to take part in the experiment, and were paid $£ 4$ for their participation. Participants were randomly allocated to one of six conditions, with sixteen in each. Participants were tested individually and were 
presented with a fully automated version of Burns and Vollmeyer's (2002) water purification system, which was run on Dell Optiplex computers.

\section{Materials and Procedure}

The present study adapted Burns and Vollmeyer's (2002) original control system as depicted in Figure 1. All participants were presented with a cover story in which they were told to imagine that they worked in a water purification plant, and that their job was to inspect the water quality of the system. The system was operated by varying the different

Figure 1. Water tank system with inputs (salt, carbon, lime) and outputs (oxygenation, chlorine concentration, temperature).

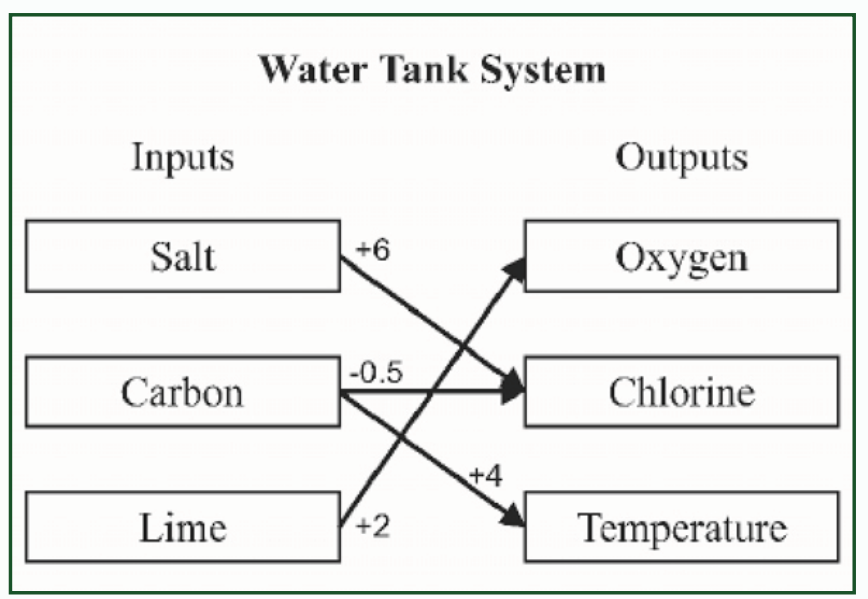

levels of salt, carbon, and lime (inputs), which then changed the three water quality indicators: oxygenation, temperature, and chlorine concentration (outputs). The CDC-task was divided into two phases, the learning phase (40 trials divided into 4 blocks of 10 trials) and the test phase, with two control tests (Control Test 1 and Control Test 2; each test was 10 trials). After every 10 trials in the learning phase, and after each control test in the test phase, all participants were presented with a structure test, consisting of a diagram of the system shown on screen, and were asked to indicate which input was connected to which output. The critical manipulations for the six conditions of this study concerned the learning phase.

Active Generate Conditions: The learning phase of both Active generate conditions (i.e., Active [generate]-Instance condition, Active [generate]-Instance + Rule condition) involved the following procedure. Each learning trial consisted of participants interacting with the system by changing any input by any value they chose, using a slider corresponding to each. Each slider had a scale from -100 to 100 units. When participants were satisfied with their changes to the inputs, they clicked a button labeled "output readings," which revealed the values of all three outputs. When they were ready to start the next trial, they clicked a button "next trial," which hid the output values from view. On the next trial, the newly changed inputs affected the output values from the previous trial: thus, the effects 
on the outputs were cumulative from one trial to the next. Prior to the learning phase, the Active (generate)-Instance condition was given a goal specific instruction. They were told that they would be required to generate input values to achieve and maintain the following output values in the system: Oxygenation $=50$, Chlorine Concentration $=700$, Temperature $=900$; these values were identical to that of Control Test 1. In addition to this, the Active (generate)-Instance + Rule condition was instructed that, at the end of each trial, they were to verbally describe the input and output changes in the form of an "if then__ statement (e.g., if input salt is changed to 10 units, then the output value of Chlorine Concentration is 516).

Active Replicate Conditions: The learning phase of the Active replicate conditions (i.e., Active [replicate]-Instance condition, Active [replicate]-Instance + Rule condition) was as follows. In contrast to participants in the Active generate condition in which they were free to choose which inputs to change and by how much on each learning trial, participants in the Active replicate conditions were required to change the inputs according to a trial history. That is, participants were presented with a sheet which contained instructions as to which input/inputs had to be change and the values they should be changed by for every trial of the learning phase. ${ }^{1}$ The Active (replicate)-Instance condition were told to simply follow the instructions on the trial history sheet and change the specific inputs by their prespecified values for each learning trial. In addition to this, the Active (replicate)Instance + Rule condition were instructed to described the input and output changes in the form of an "if then "statement.

Observe Conditions: The learning phase of both Observe conditions (i.e., ObserveInstance condition, Observe-Instance + Rule condition ) was as follows: Instead of changing the inputs as in the active learning-based conditions, on each trial participants pressed a button "reveal inputs," then observed the sliders of the inputs changing automatically according to prespecified values. Then they pressed a button "reveal outputs," which displayed the corresponding effects on the output values. After studying them, participants clicked a button "ready for next trial," which cleared the input and output values ready for the next trial.In the Observe-Instance condition, participants'job was to carefully track the changes to the inputs and outputs on each trial, which changed according to the same trial history presented in the active-replicate conditions, and to assess how successfully the output values met the criteria output values. In addition to this, the Observe-Instance + Rule condition were told to describe the input and output changes in the form of an "if___ then___ statement.

After the learning phase, participants were presented the test phase.The procedure in the test phase was the same for all six conditions. The criterion values participants had to reach and maintain in Control Test 1 were Oxygenation $=50$, Chlorine Concentration = 700 , Temperature $=900$, and in Control Test 2 the values were Oxygenation $=250$; Chlorine Concentration $=350$; Temperature $=1100$. The output criteria in Control Test 2 were un- 
familiar to all six conditions, since this control test involved a goal that they had not been trained on, and provided a test of the generalizability of their control skills.

On completion of the experiment, participants were presented with a record of three different trial histories from the learning phase: one that they had actually experienced during the learning phase, and two randomly selected alternatives from the Active (generate) conditions. They were asked to decide which of the three they had experienced, and what formed the basis of their judgment: that is, did they recognize specific trials? Did they rely on a sense of familiarity? Did they guess?

\section{Scoring}

Structure test performance was based on computing the proportion of input-output links correctly identified for each test. A correction for guessing was incorporated: correct responses (i.e., the number of correct links included, and incorrect links avoided), incorrect responses (i.e., the number of incorrect links included, and correct links avoided)/ N (the total number of links that could be made). The maximum value for each structure score was 10 . Successful performance is indicated by higher structure test scores.

\section{Control Tests 1 and 2}

Control performance was measured as error scores, and was calculated in the same way for each test. Error scores were based on calculating the difference between each target's output value (i.e., the criterion according to the test) and the actual output value produced by the participant for each trial of the transfer test. A log transformation (base 10) was applied to the error scores of each participant for each trial, to minimize the skewedness of the distribution of scores. All analyses of error scores are based on participants' mean error, averaged over all 10 trials, across all three output variables. Successful control performance is indexed by the difference between the achieved and target output values, thus lower error scores indicate better performance.

\section{Results}

\section{Test of Control Skills}

The mean error score of all six conditions presented in Figure 2 suggests that, in all three Instance + Rule-based conditions, control error scores were lower (indicating good performance) in both tests, compared with the three Instance only-based conditions.

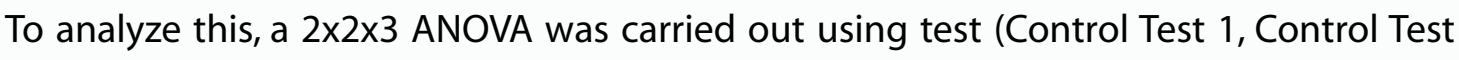
2) as within subject variables, and knowledge (Instance, Instance + Rule) and learning format (Active [generate], Active [replicate], Observe) as the between subject variables. The analysis showed a significant main effect of test: $F(1,90)=4.87, \mathrm{MSE}=0.13, p<0.05$, 
Figure 2. Mean Control Test error scores $( \pm S E)$ at Control Test 1 and Control Test 2 for each condition. Successful performance is indicated by lower mean error scores.

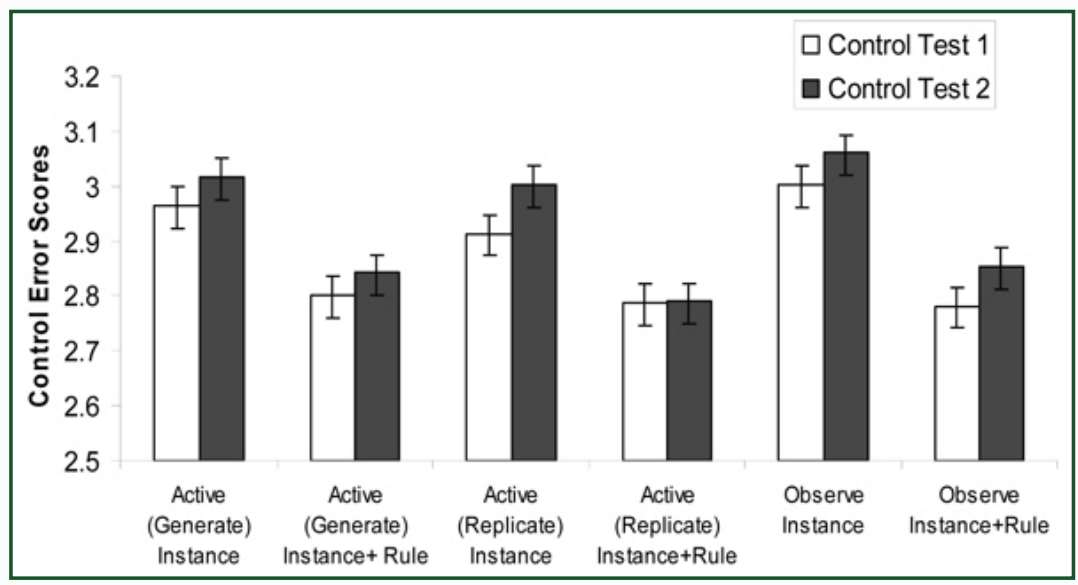

$\eta^{2}=0.05$. There was also a significant main effect of knowledge: $F(1,90)=59.22$, MSE $=$ $1.56, p<0.005, \eta^{2}=0.39$. No other main effects or interactions were significant. Because there was no interaction between knowledge and test, the control error scores for each condition were collapsed across tests. The significant higher control error scores (impaired performance) in the Instance conditions compared with the Instance + Rule conditions for the Active (generate), Active (replicate), and Observe conditions, was confirmed by a planned comparison for error scores: $\mathrm{t}(15)=2.75, p<0.05, d=1.42, \mathrm{t}(15)=5.05, p<0.05$, $d=2.61$, and $\mathrm{t}(15)=8.78, p<0.005, d=4.53$, respectively.

Test of rule-based knowledge: The mean structure test scores were collapsed across phase for each of the six conditions and are presented in Figure 3. The figure suggests

Figure 3. Mean Structure test scores $( \pm S E)$ averaged across the learning phase and again for the Control test phase for each condition. Successful performance is indicated by higher structure scores.

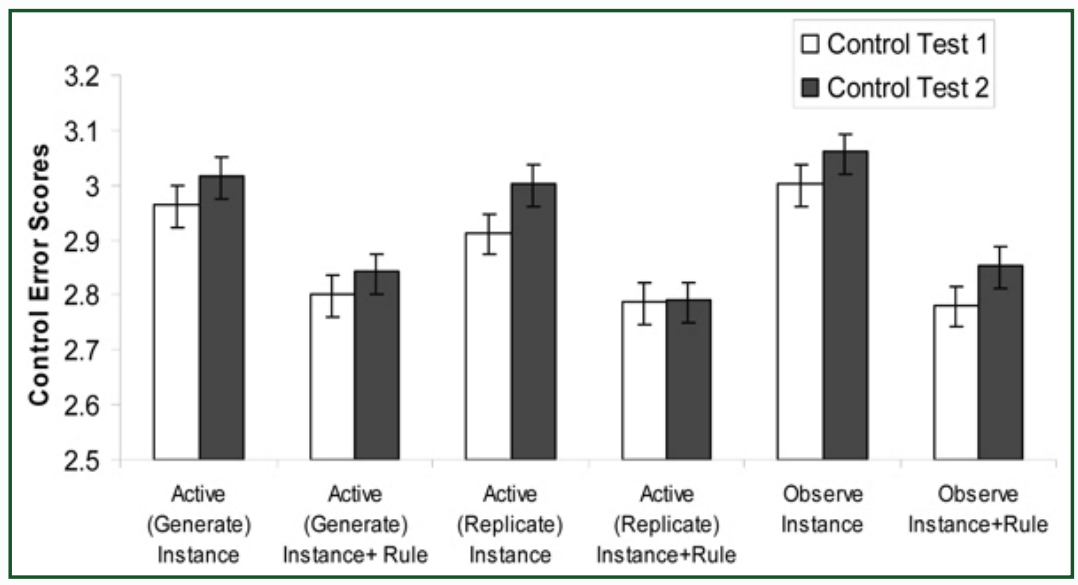

that, in the three Instance + Rule-based conditions, structure test scores were lower (indicating good performance) compared with the three Instance only-based conditions. To 
analyze this, a $2 \times 2 \times 3$ ANOVA was carried out using phase (Learning, Test) as within subject variables, and knowledge (Instance, Instance + Rule) and learning format (Active [generate], Active [replicate], Observe) as the between subject variables. The analysis showed a significant main effect of phase: $F(1,90)=4.561$, MSE $=0.18, p<0.05, \eta^{2}=0.48$. There was also a significant main effect of knowledge: $F(1,90)=29.58, \mathrm{MSE}=4.18, p<0.0005, \eta^{2}=0.24$. No other main effects or interactions were significant. Because there was no interaction between knowledge and test, the structure test scores for each condition were collapsed across phase. Planned comparisons confirmed higher structure test scores (improved performance) in the Instance + Rule conditions, compared with the Instance conditions for the Active (generate), Active (replicate), and Observe conditions: $\mathrm{t}(15)=2.65, p<0.05$, $d=1.37, \mathrm{t}(15)=3.19, p<0.01, d=1.65$, and $\mathrm{t}(15)=4.37, p<0.001, d=2.26$, respectively.

Correlation between control performance and structural knowledge. The following correlation analyses were carried out on control error scores (averaged across Control Tests 1-2), and structure test scores (averaged across Structure Tests 5-6 in the test phase). Scores were collapsed across the four active conditions, and then again for the remaining observation conditions. Correlation analyses revealed a significant negative relationship between structure test scores and control test scores: $r(64)=-0.31, p<0.05$, and, $r(32)=$ $-0.47, p<0.01$, respectively.

Test of self-insight. Table 1 indicates that more correct identifications of the learning trials experienced during the learning phase were made in Instance + Rule conditions than in Instance only conditions. A Chi-squared analysis, collapsing responses across

Table 1. Mean Control Test error scores $( \pm S E)$ at Control Test 1 and Control Test 2 for each condition. Successful performance is indicated by lower mean error scores.

\begin{tabular}{lcccc|}
\hline & \multicolumn{4}{c}{ Basis for Identification } \\
\hline Condition & $\begin{array}{c}\text { No. of Correct } \\
\text { Identifications }\end{array}$ & $\begin{array}{l}\text { Feeling of } \\
\text { Familiarity }\end{array}$ & $\begin{array}{c}\text { Recognize } \\
\text { specific } \\
\text { instances }\end{array}$ & Guess \\
& & 5 & 5 & 6 \\
Active (Generate) Instance & 5 & 6 & 8 & 2 \\
Active (Generate) Instance + Rule & 9 & 5 & 4 & 7 \\
Active (Replicate) Instance & 4 & 3 & 8 & 5 \\
Active (Replicate) Instance + Rule & 10 & 6 & 3 & 7 \\
Observe Instance & 4 & 5 & 9 & 2 \\
Observe Instance + Rule & 9 & & & \\
\hline
\end{tabular}

action-based conditions, and comparing accuracy of responding in Instance and Instance + rule conditions, confirmed the trend indicated in Table $1: \chi(3)=8.78, p<0.05$. Furthermore, Table 1 suggests that more participants relied on specific instances to identify their learning phase in Instance + Rule conditions than in Instance conditions, whereas the Instance-based conditions relied more on guessing. This was also confirmed using a Chi-squared analysis: $\chi(3)=9.47, p<0.05$. 


\section{Discussion}

The findings from this study can be summarized as follows: First, the evidence shows that the generation of instance-based knowledge in conjunction with rule-based knowledge leads to better control performance and accurate structural knowledge of the task, compared to conditions in which only instance-based knowledge was acquired during learning. Second, consistent with previous studies (Osman 2008a, 2008b), there was no advantage of active-based learning over observation-based learning in both measures of performance.Third, also consistent with previous studies (Burns \& Vollmeyer, 2002; Osman 2008a, 2008b; Sanderson, 1989), the present study revealed an association between the accuracy of participants' knowledge of the structure of the system they were controlling, and their ability to control it. Fourth, posttest questions indicated that when identifying the learning trials they experienced during the learning phase from two others, participants were more accurate in conditions in which the learning phase generated instance and rule-based knowledge compared with instance only conditions.

The target question that was asked in this article considered the following: Is procedural learning necessary to ensure skill acquisition in a complex dynamic environment? Overall, the evidence from this study shows that procedural learning is sufficient for the successful uptake of relevant knowledge, but not necessary, given that observational learning produced patterns of performance equivalent to those of the active learning conditions. Implicit learning theorists (Berry, 1991; Berry \& Broadbent, 1988; Sun et al., 2001) have maintained that the knowledge required to control CDC-tasks is procedural based, and embedded within the interactions problem solvers have with the system. Furthermore, knowledge is conscious only to the extent that the response that is appropriate to a given situation can be stated, but the knowledge used to support that response is unavailable to consciousness (Buchner et al., 1995; Dienes \& Berry, 1997; Dienes \& Fahey, 1998). Similar claims are made for other tasks considered to be procedural learning tasks, or called, alternatively, implicit learning tasks (e.g., artificial grammar learning, Reber, 1989; sequence learning, Nissen \& Bullemer, 1987; Willingham et al., 2000). While CDC-tasks are typically classed as implicit learning environments, the findings from the present study, along with previous studies ( Burns \& Vollmeyer, 2002; Brehmer, 1992; Gibson et al., 1997; Schoppek, 2002; Vollmeyer et al, 1996), challenge this classification. The evidence from the present study shows that rule-based knowledge was in fact associated with procedural knowledge and lead to better performance on measures of procedural knowledge. Moreover, the present study is the first of its kind to provide clear evidence that explicitly thinking about the relationship between events and outcomes as rules leads to superior knowledge about how a CDC-task works, how to operate it, how to transfer control skills to an untrained goal, and self-insight into what one does to learn about how to operate it. 
Why were associations found between declarative and procedural knowledge?

Typically studies using CDC-tasks in which dissociations are usually reported in studies in which structural knowledge of the task is examined only after learning takes place (Berry, 1991; Berry \& Broadbent, 1984, 1987; 1988; Broadbent et al. 1986; Dienes \& Fahey, 1995; 1998; Marescaux et al., 1989). Without an opportunity to keep track of ones knowledge of the rule or structure the system operates under, explicit knowledge is found to be poor (Burns \&Vollmeyer, 2002; Sanderson, 1989; Sanderson \& Vicente, 1986). In contrast to this, demonstrations of associations between procedural and declarative knowledge are reported in studies that encourage hypothesis-testing behaviors during learning (Burns \& Vollmeyer, 2002; Gonzales \& Quesada, 2003; Jensen \& Brehmer, 2003; Sweller, 1988).In addition, indices of both types of knowledge reveal that performance exceeds that of conditions in which hypothesis testing is prevented (Burns \& Vollmeyer, 2002; Sweller, 1988; Vollmeyer et al., 1996). Consistent with this, the present study included both types of methods designed to uncover associations between procedural and declarative knowledge. Participants had regular opportunities to examine their structural knowledge of the system during knowledge acquisition (both in active and observation-based learning) and those in the Instance + Rule conditions were encouraged to hypothesis test as a result of verbalizing their knowledge in the form of conditional statements.

The findings from this study converge with previous evidence suggesting that instructions designed to encourage hypothesis testing and other similar meta-cognitive processes (e.g., monitoring-tracking one's online goal-directed behaviors) do not interfere with the uptake of skilled knowledge, as some have claimed, but instead enhance skilled performance (Berardi-Coletta et al., 1995; Osman, 2008a, 2008b). Thus, the present study shows that hypothesis testing, rather than the active engagement with a procedural task, is necessary for the successful uptake of knowledge and its application to mastering a complex control system.

\section{Acknowledgements}

Preparation for this article was supported by Economic and Social Research Council ESRC grant RES-000-27-0119.The support of the Economic and Social Research Council (ESRC) is gratefully acknowledged.The work was also part of the programme of the ESRC Research Centre for Economic Learning and Human Evolution.

\section{Note}

1. The trial histories for the Active (replicate-instance), Active (replicate-Instance + Rule), Observe (Instance), and Observe (Instance + Rule) conditions were based on the learning phase generated by one of the participants from the Active (generate - Instance + Rule) condition. The participant was selected on the basis of their control performance in the test phase, which was closest to the mean 
across both Active (generate - instance) and Active (generate - Instance + Rule) conditions. This was favored instead of a full yoking procedure because the source of any differences in these conditions was carefully controlled, and was likely to result from the instructional manipulations during learning, rather than from the trial history itself.

\section{References}

Anderson, J. R. (1982). Acquisition of cognitive skill. Psychological Review, 89, 369-406.

Berardi-Coletta, B., Dominowski, R., Buyer, S., \& Rellinger, E. (1995). Metacognition and problem solving: A Process orientated approach. Journal of Experimental Psychology: Learning, Memory, \& Cognition, 21, 205-223.

Berry, D. (1991). The role of action in implicit learning. Quarterly Journal of Experimental Psychology, 43, 881-906.

Berry, D., \& Broadbent, D. E. (1984). On the relationship between task performance and associated verbalizable knowledge. Quarterly Journal of Experimental Psychology, 36, 209-231.

Berry, D., \& Broadbent, D. E. (1987). The combination of implicit and explicit knowledge in task control. Psychological Research, 49, 7-15.

Berry, D.C., \& Broadbent, D. E. (1988). Interactive tasks and the implicit-explicit distinction. British Journal of Psychology, 79, 251-272.

Brehmer, B. (1992). Dynamic decision making: Human control of complex systems. Acta Psychologica, 81, 211-241.

Broadbent, D., Fitzgerald, P., \& Broadbent, M.H.P.(1986). Implicit and explicit knowledge in the control of complex systems. British Journal of Psychology, 77, 33-50.

Buchner, A., Funke,J.,\& Berry,D. (1995). Negative correlations between control performance and verbalizable knowledge: Indicators for implicit learning in process control tasks. Quarterly Journal of Experimental Psychology, 48, 166-187.

Burns, B.D., \&Vollmeyer, R. (2002). Goal specificity effects on hypothesis testing in problem solving. Quarterly Journal of Experimental Psychology, 55, 241-261.

Dienes,Z., \& Berry, D. (1997).Implicit learning:Below the subjective threshold.Psychonomic Bulletin \& Review, 4, 3-23.

Dienes, Z., \& Fahey, R. (1995). Role of specific instances in controlling a dynamic system. Journal of Experimental Psychology: Learning, Memory, \& Cognition, 21, 848-862.

Dienes, Z., \& Fahey, R. (1998). The role of implicit memory in controlling a dynamic system. Quarterly Journal of Experimental Psychology, 51,593-614.

Gibson,F.P., \& Fichman, M., \& Plaut,D.C.(1997).Learning in dynamic decision tasks:Computational model and empirical evidence. Organizational Behavior and Human Decision Processes, 71, 1-35.

Gonzales, C., \& Quesada, J. (2003). Learning in dynamic decision making:The recognition process. Computational \& Mathematical Organization Theory, 9, 287-304. 
Jensen, E., \& Brehmer, B. (2003). Understanding and control of a simple dynamic system. Systems Dynamic Review, 19, 119-137.

Lee, Y. (1995). Effects of learning contexts on implicit and explicit learning. Memory and Cognition, 23, 723-734.

Nissen, M. J., \& Bullemer, P. (1987). Attentional requirements of learning-evidence from performance-measures. Cognitive Psychology, 19, 1-32.

Osman, M. (2008a). Observation can be as effective as action in problem solving. Cognitive Science, 32, 162-183.

Osman, M. (2008b). Evidence for positive transfer and negative transfer/Anti-learning of problem solving skills. Journal of Experimental Psychology: General, 137, 97-115.

Reber, A. S. (1989). Implicit learning and tacit knowledge. Journal of Experimental Psychology, 118, 219-235.

Sanderson,P.M.(1989).Verbalizable knowledge and skilled task performance:Association, dissociation, and mental models.Journal of Experimental Psychology: Learning, Memory, \& Cognition, 15, 729-747.

Sanderson, P.M., \& Vicente, K.J. (1986). Verbalizable knowledge and skilled task performance: Explaining association and dissociation (Technical Report EPL-86-04).Urbana, IL:University of Illinois at Urbana-Champaign. Engineering Psychology Research Laboratory.

Schoppek, W. (2002). Examples, rules and strategies in the control of dynamic systems. Cognitive Science Quarterly, 2, 63-92.

Stanley, W. B., Mathews, R. C., Buss, R. R., \& Kotler-Cope, S. (1989). Insight without awareness: On the interaction of verbalization, instruction, and practice in a simulated process CDCT. Quarterly Journal of Experimental Psychology, 41, 553-577.

Sun, R., Merrill,E., \& Peterson, T. (2001).From implicit skills to explicit knowledge:A bottomup model of skill learning. Cognitive Science, 25, 203-244.

Sweller, J. (1988). Cognitive load during problem solving: Effects of learning. Cognitive Science, 12, 257-285.

Vollmeyer, R., Burns, B. D., \& Holyoak, K. J. (1996). The impact of goal specificity and systematicity of strategies on the acquisition of problem structure. Cognitive Science, 20, 75-100.

Willingham, D. B.,Wells, L. A., Farrell, J.M., \& Stemwedel, M.E.(2000).Implicit motor sequence learning is represented in response locations. Memory \& Cognition, 28, 366-375.

Paper submitted on Sep 21, 2007.

Final version accepted on Dec 9, 2007. 